\title{
On cosmological mass with positive $\Lambda$
}

\author{
Roger Penrose
}

Received: 12 July 2011 / Accepted: 21 July 2011 / Published online: 19 August 2011

(C) The Author(s) 2011. This article is published with open access at Springerlink.com

\begin{abstract}
For asymptotically flat space-times, a very satisfactory expression for the total mass/energy of a system defined at future null infinity was provided by Bondi and Sachs, in the early 1960s. A generalization of this to space-times that are asymptotically de Sitter now has particular relevance in view of observational evidence, from 1998 onwards, indicating the presence of a positive cosmological constant $\Lambda$. In this article, some of the issues involved in such a definition are examined, showing that a somewhat different attitude to mass/energy must be taken, from that which was appropriate for asymptotically flat space-times. Two tentative suggestions are put forward for a retarded mass/energy definition with positive $\Lambda$, one based on a conformally invariant integral expression whose advanced time-derivative gives the Bondi-Sachs definition in the asymptotically flat case and the other, on the author's 1982 approach to quasi-local energy. Such expressions could have some direct relevance to the proposal of Conformal Cyclic Cosmology, for which recent analysis of the CMB has provided some striking support.
\end{abstract}

Keywords Cosmological constant - Energy in cosmology · Bondi-Sachs mass . Conformal cyclic cosmology

\section{The role of positive $\Lambda$}

It is a great pleasure for me to have this opportunity to pay my respects to my old friend Joshua Goldberg in honour of his 86th birthday. I hope that the ruminations presented here, incomplete as they are, may provide at least a partial indication of the

\author{
R. Penrose $(\varangle)$ \\ Mathematical Institute, 24-29 St Giles', Oxford OX1 3LB, UK \\ e-mail: penroad@herald.ox.ac.uk \\ R. Penrose \\ Department of Physics, University of Leiden, 2 Niels Bohrweg, Leiden, The Netherlands
}


admiration and warm regard that I have for Josh, not only as a person, but in view of his great interest in, and powerful contributions to, conservation laws in general relativity [1,2]. I am concerned, in this article, with a notion of mass/energy aimed to apply to Einstein's general relativity when there is a positive cosmological constant $\Lambda$ present.

Clear evidence for the actual physical presence of a positive $\Lambda$ (or possibly of some kind of alternative "dark-energy" field that mimics such a $\Lambda$-term in Einstein's theory) originated from the independent observations of distant supernovae made by the teams headed by Saul Permutter and by Brian Schmidt in 1998 [3], and has been subsequently re-enforced by further work. ${ }^{1}$ On the basis of this finding, I proposed, in 2005, the cosmological scheme referred to as "conformal cyclic cosmology" (abbreviated CCC) which depends crucially on the actual existence of a positive $\Lambda$ (see Penrose [5]). The cosmological picture that is presented by current theory and observation asserts that our universe originated with Big-Bang event and has been expanding ever since, the $\Lambda$-term in Einstein's equations telling us that this expansion should continue exponentially to eternity. CCC proposes that this is only a partial picture, and that the entire history of the universe as presented by current theory is but one "aeon" in a continuing succession of such aeons, the conformal infinity of each matching exactly the conformally "stretched" geometry of the big bang. ${ }^{2}$ The very early inflationary phase to the universe that is normally regarded as an essential part of standard cosmology is also eliminated in $\mathrm{CCC}$, the final exponential expansion of the previous aeon serving all the purposes for which inflation appears to be needed.

In a recent arXiv note, Gurzadyan and Penrose [6] presented significant evidence in support of one of the predicted effects of CCC, namely signals within the CMB that would result from encounters between supermassive black holes in the aeon prior to our own. According to $\mathrm{CCC},{ }^{3}$ repeated gravitational wave bursts from these encounters within a single galactic cluster in the previous aeon ought to result in sets of concentric circles in the CMB sky of notably lower temperature variance than in the general background. In this arXiv note, we find that in an examination of the WMAP data for points in the whole sky (excluding only the galactic plane region) which are centres of at least three such low-variance circles, there are 352 such centers. In order to estimate the likelihood that this number could have arisen merely from chance fluctuations, we repeated exactly this same analysis using a standard type of simulation of the CMB sky (employing the observed WMAP power spectrum-i.e. 1-value of a spherical-harmonic analysis_-together with a randomization of the m-values $)^{4}$

\footnotetext{
1 For example, see Carroll [4].

2 I here use the capitalized "Big Bang" for the particular event that originated our present aeon. The term "big bang" refers, more generally to the event of origin of some unspecified aeon of the sequence.

3 See Penrose [5], Section 3.6.

4 In view of criticisms from others (e.g. Werhus and Eriksen [7], Moss et al. [8]) to a previous note that we had put on the arXiv, in which the contrast between the simulation and the observed WMAP data was even more pronounced, we here adopted a standard procedure for constructing the simulations-basically identical to those used by our critics. This is not to be taken as an indication that we agree that the statistical procedures adopted by our critics is the correct one, but it is allowing them the benefit of the doubt in this particular analysis in order to demonstrate the considerable strength of our case, even when their procedures for analysis are adopted.
} 
finding only 79 centres in the simulation. As this was the only simulation that we had analyzed in this way we repeated the procedure with another such simulation, again chosen randomly, this time finding only 47 such centres. At the time of writing, no other group has repeated our analysis, either to confirm or refute what we have done. In the absence of any such refutation, it seems that a plausible pointer has been made in the direction of establishing CCC as providing a significant contribution to cosmological truth.

An important ingredient of the theoretical arguments underlying $\mathrm{CCC}$ would be a clear-cut definition of the appropriate notion of "mass/energy" transported by gravitational waves in cosmological models in which there is a positive $\Lambda$. This would appear to be an essential pre-requisite for ascertaining the transfer of energy carried by gravitational waves from the supermassive black-hole encounter in the previous aeon to the disturbance in the initial material in our own aeon's Big Bang, as predicted by CCC. We shall see in this article that a number of new issues are raised, concerning the question of energy conservation, that do not appear to have been adequately addressed before. Since we do not have asymptotic flatness, when $\Lambda>0$, the accepted (Bondi-Sachs) notions of energy carried by gravitational waves cannot be used, the space-time geometry being (in an appropriate sense) asymptotically de Sitter rather than asymptotically flat.

\section{Bondi-Sachs mass/energy}

The early days of general relativity, the fact that gravitational energy could not be localized in space-time, in a coordinate-independent way had been disturbing to many theorists, and often regarded as a reason for being suspicious of Einstein's theory (see e.g. Pais [9]). Einstein's own approach to this issue had been to assign a "pseudotensor" to represent gravitational energy whose coordinate-dependent nature reflected gravitational energy's non-local character. This indicated that one could only talk precisely about the gravitational contribution to the energy of a system in terms of an asymptotic limit, taken out to infinity. With a brief note to Nature in 1960 [10], Hermann Bondi put forward the essential basis of his own idea for defining the total retarded mass/energy of a system, by adopting a coordinate system in which a retarded time coordinate was used, chosen to be constant on a family of outgoing null hypersurfaces. (Somewhat similar ideas had been put forward earlier by Trautman in 1958 $[11,12]$.) Bondi had made the additional simplifying assumption of axi-symmetry, and developed the formalism in this framework in considerable detail with van der Berg and Metzner in 1962 [13]. Very shortly afterwards, Rainer Sachs [14,15], generalized this work, removing the restriction of axi-symmetry and exploring the relations to asymptotic symmetry, so that the asymptotic measure of total momentum could also be identified (see Bonnor and Rotenberg [16]).

The advantage of using a notion of retarded time in this definition was that it became possible to identify the contribution to the total mass/energy that is carried away from an isolated system by gravitational radiation. Bondi introduced the term "news function" for the quantity-denoted here by $\mathfrak{N}$-whose absolute square $\mathfrak{N} \overline{\mathfrak{N}}$ integrated over the sphere at infinity, measures the rate of energy loss in gravitational radiation. In Bondi's original axi-symmetrical situation, $\mathfrak{N}$ was a real number, but in 
Sach's extension to the general case, $\mathfrak{N}$ became a complex (spin-weighted) quantity defined at future-null infinity.

Various other procedures for addressing the issue were developed by others [2,17-19], but my own particular geometrical approach (Penrose [20-22], Penrose and Rindler [24], Chapter 9.) involved introducing a conformal rescaling of the physical metric $\hat{g}_{a b},{ }^{5}$ so that future-null infinity $\mathcal{I}^{+}$becomes a null-hypersurface boundary (henceforth denoted simply by $\mathcal{I}$, since no past conformal infinity is considered in this article) which is attached smoothly to space-time, where the rescaled metric

$$
g_{a b}=\omega^{2} \hat{g}_{a b}
$$

can be continued smoothly to (and even beyond) the hypersurface $\mathcal{I}$. Here I use the notation " $\omega$ " for the conformal factor, which becomes zero smoothly at $\mathcal{I}$ as it is crossed, having a non-vanishing future-pointing derivative there (so as to be consistent with the notation used in Appendix B of Cycles of Time [5]). Accordingly, $\omega$ is taken to be negative within the physical space-time $\mathcal{M}$, but the inverse quantity

$$
\Omega=-\omega^{-1}
$$

is positive within $\mathcal{M}$, becoming infinite at $\mathcal{I}$. We now have, for the physical metric $\hat{g}_{a b}$,

$$
\hat{g}_{a b}=\Omega^{2} g_{a b}
$$

For an asymptotically flat $\mathcal{M}$, the future conformal infinity $\mathcal{I}$ is a null hypersurface, but for $\mathcal{M}$ with a positive cosmological constant $\Lambda$, this hypersurface $\mathcal{I}$ is spacelike $[20,21]$. This feature is essential for CCC, since in that scheme $\mathcal{I}$ needs to be matched conformally to a spacelike big-bang hypersurface $\mathcal{B}$, where $\mathcal{B}$ is taken to be a smooth past boundary to the succeeding aeon which, in accordance with a particular mathematical proposal for formulating the "Weyl curvature hypothesis" introduced by Tod [25] in 2003, ensures that the gravitational degrees of freedom are hugely suppressed at that aeon's big bang, as appears to be the case observationally for the Big Bang of our own aeon.

In the conformal approach to defining the Bondi-Sachs mass/energy, we first note that there is an important discrepancy between certain conformal scalings. On the one hand, we have the invariance under $\hat{g}_{a b}=\Omega^{2} g_{a b}$ given by

$$
\hat{C}_{a b c}^{d}=C_{a b c}{ }^{d} \text { and } \hat{\Psi}_{A B C D}=\Psi_{A B C D},
$$

where $C_{a b c d}$ is the Weyl conformal curvature tensor and $\Psi_{A B C D}$ is its spinor equivalent, these two quantities being related to each other by

$$
C_{a b c d}=\Psi_{A B C D} \varepsilon_{A^{\prime} B^{\prime}} \varepsilon_{C^{\prime} D^{\prime}}+\varepsilon_{A B} \varepsilon_{C D} \bar{\Psi}_{A^{\prime} B^{\prime} C^{\prime} D^{\prime}}
$$

\footnotetext{
5 Throughout this article I use abstract indices, consistently with the notation of Penrose and Rindler $[23,24]$.
} 
(abstract-index notation, signs, etc., as in Spinors and Space Time [23]), $C_{a b c d}=$ $C_{[c d][a b]}, C_{[a b c] d}=0, C_{a b c} a=0, \Psi_{A B C D}=\Psi_{(A B C D)}$. On the other hand, we may want to have a tensor $K_{a b c d}$ or its spinor equivalent $\psi_{A B C D}$, related to each other in exactly the same way

$$
K_{a b c d}=\psi_{A B C D} \varepsilon_{A^{\prime} B^{\prime}} \varepsilon_{C^{\prime} D^{\prime}}+\varepsilon_{A B} \varepsilon_{C D} \bar{\psi}_{A^{\prime} B^{\prime} C^{\prime} D^{\prime}}
$$

and taken to be proportional, respectively, to $C_{a b c d}$ and to $\Psi_{A B C D}$, but where under $\hat{g}_{a b}=\Omega^{2} g_{a b}$ we demand

$$
\hat{K}_{a b c}^{d}=\Omega^{-1} K_{a b c}{ }^{d} \text { and } \hat{\psi}_{A B C D}=\Omega^{-1} \psi_{A B C D}
$$

The reason for this different scaling is to achieve conformal invariance (in vacuum) for the propagation equations (the spin-2 massless "Schrödinger equations for gravitons"):

$$
\nabla_{d} K_{a b c}^{d}=0\left(\text { i.e. } \nabla_{[e} K_{a b] c d}=0\right) ; \text { equivalently } \nabla^{A A^{\prime}} \psi_{A B C D}=0
$$

In the physical metric $\hat{g}_{a b}$ we demand the equalities

$$
\hat{C}_{a b c}^{d}=\hat{K}_{a b c}^{d} \text { and } \hat{\Psi}_{A B C D}=\hat{\psi}_{A B C D},
$$

but the discrepancy is revealed when we pass to the metric $g_{a b}$, for which the conformal boundary $\mathcal{I}$ has been introduced.

This conformal invariance of the propagation equations is a key ingredient of the conformal approach to the study of radiation fields, because it tells us that the radiation propagates to (and "through") $\mathcal{I}$ just as it would at any finite hypersurface, not really "noticing" that $\mathcal{I}$ actually represents infinity. The radiation field registers its presence by attaining finite values at $\mathcal{I}$. The same holds for electromagnetic radiation, and also for the quantities that are needed for the determination of the total mass/energy of a system as measured at some specific retarded time, this retarded time being specified by some family of cross-sections - or "cuts" $\mathcal{S}$ - of $\mathcal{I}$. The difference in scaling behavior between $\Psi_{A B C D}$ and $\psi_{A B C D}$ (or, equivalently, between $C_{a b c}$ and $K_{a b c}{ }^{d}$ ) leads to the conclusion that $\Psi_{A B C D}$ (and $C_{a b c}$ ) must vanish at $\mathcal{I}$ and that it is normal derivative of these conformal curvature quantities across $\mathcal{I}$ that provides us with the measure of gravitational radiation, and related quantities.

It is usual to refer the relevant spinor quantities on $\mathcal{I}$ to a spin-frame $\left(o^{A}, \iota^{A}\right)$, where the flagpole of $\iota^{A}$ points along the (null) generators of $\mathcal{I}$, and where that of $o^{A}$ points along the other null normal to each $\mathcal{S}$ of the chosen family i.e. along the (null) generators of the hypersurfaces $\mathcal{N}$ of constant retarded time. The quantity that must be integrated over $\mathcal{S}$ in order to define the Bondi-Sachs mass, at the retarded time specified by $\mathcal{S}$, in the conformal approach, is

$$
\mu_{B S}=\frac{1}{4 \pi G}\left(\sigma \mathfrak{N}-\psi_{2}\right)
$$


where $\psi_{2}$ is the component

$$
\psi_{2}=\psi_{0011}=\psi_{A B C D} O^{A} o^{B}{ }_{\iota}{ } \iota^{D}
$$

on the cut $\mathcal{S}$, where $\mathcal{N}$ meets $\mathcal{I}$. The quantity $\sigma$ is the complex shear

$$
\sigma=o^{A} o^{B} \bar{\iota}^{A^{\prime}} \nabla_{A A^{\prime}} o_{B}
$$

of the generators of $\mathcal{N}$ (at $\mathcal{I}$ ), and the quantity $\mathfrak{N}$ is the Bondi-Sachs news function

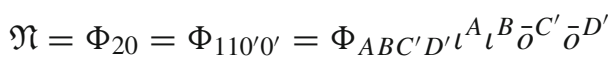

on $\mathcal{I}$, where $\Phi_{A B C^{\prime} D^{\prime}}$ is essentially the trace-free Ricci tensor

$$
R_{a b}-\frac{1}{4} R g_{a b}=-2 \Phi_{A B A^{\prime} B^{\prime}}
$$

Now, suppose that $\mathcal{S}_{1}$ and $\mathcal{S}_{2}$ are two cuts of $\mathcal{I}$, where $\mathcal{S}_{2}$ lies entirely to the future of $\mathcal{S}_{1}$ along $\mathcal{I}$. Suppose, also, that the conformal scaling at $\mathcal{I}$ is chosen so that the intrinsic metric of every cut is that of a unit sphere. The Bondi-Sachs mass-loss formula tells us that the integral of $\mu_{B S}$ over $\mathcal{S}_{1}$, with respect to surface area, is never smaller than the corresponding integral of $\mu_{B S}$ over $\mathcal{S}_{2}$, and that the difference is equated to the integral over the region of $\mathcal{I}$ between $\mathcal{S}_{1}$ and $\mathcal{S}_{2}$, of $(4 \pi G)^{-1} \mathfrak{N} \overline{\mathfrak{N}}$ plus any contribution from the energy momentum tensor of massless radiation that may have crossed $\mathcal{I}$ between $\mathcal{S}_{1}$ and $\mathcal{S}_{2}{ }^{6}$

\section{New issues arising from a positive $\Lambda$}

The case of a positive $\Lambda$ presents us with several new issues, mainly to do with the spacelike nature of $\mathcal{I}$, and the main purpose of this article is to point these out. I do not have a full resolution of all these matters, but some suggestions will be made in Sect. 4.

The first point of difficulty to consider is that the very notion of "energy" is problematic when there is a cosmological constant in Einstein's equations. I shall be concerned here only with the cosmologically clearly relevant case, which is general relativity with $\Lambda>0$. (This is despite the great current interest in the "AdS/CFT conjecture", see [26,27] for which $\Lambda$ is taken to be negative.) When $\Lambda>0$, we are concerned with asymptotically de Sitter space-times, rather than the asymptotically flat ones of Sect. 2. The problematic nature of the energy notion is made especially evident when we consider that even if we are concerned only with very weak gravitational fields, these would be perturbations of de Sitter space $\mathfrak{D}$, rather than of Minkowski space $\mathcal{M}$. There is no appropriate analogue of the subgroup of motions of $\mathcal{M}$ that

\footnotetext{
6 Most derivations of the Bondi-Sachs mass-loss formula seem to require that $S_{2}$ is an asymptotic timetranslation of $S_{1}$ (see Sachs [15]). As far as I am aware, a proof of the more general statement provided here was first enunciated in Penrose [22]; see also Penrose and Rindler [24], Section 9.10.
} 
is associated with energy-momentum, namely its 4-dimensional translation group. Moreover, there is not even any globally defined timelike Killing vector in $\mathfrak{D}$, which would seem to be required for an adequate notion of energy to be defined-even in the completely gravity-free case.

This last problem is only partially addressed if we restrict attention to an appropriate subspace $\mathfrak{D}^{\prime}$ of $\mathfrak{D}$. It might be thought that such a subspace would be that which is relevant to the old steady-state model of the expanding universe, since "steady state" means "forever unchanging", so there should be a Killing vector describing the unchanging nature of this model. Indeed, this is the case, but this stationary model possesses a cosmological event horizon, outside which the chosen Killing field becomes spacelike (so that the galaxies are described as exceeding the speed of light from the perspective of this Killing field). The region within which the Killing vector remains timelike converges on only a single point of the spacelike $\mathcal{I}$.

We can get a good picture of this situation by examining the representation ${ }^{7}$ of de Sitter space $\mathfrak{D}$ as the 4-hyperboloid (with two spatial directions suppressed)

$$
-t^{2}+w^{2}+x^{2}+y^{2}+z^{2}=3 / \Lambda
$$

in Minkowski 5-space $\mathcal{M}^{5}$ with standard coordinates $(t, w, x, y, z)$. The steady-state portion is taken to be that part of the hyperboloid for which $t>w$. The integral curves of this Killing vector would be the lines of intersection of the hyperboloid with the timelike 2-planes given by

$$
x=\text { const }, \quad y=\text { const }, \quad z=\text { const },
$$

and these lines remain timelike only when $x^{2}+y^{2}+z^{2}<3 / \Lambda$. All these world-lines converge on a single point of $\mathcal{I}$.

The situation is happier if we ask only for conformal Killing vectors, for the orthogonal trajectories to the spacelike 3-surfaces of "constant steady-state time", namely the intersections of the hyperboloid with the 4-surfaces

$$
t-w=\text { const }>0 .
$$

These curves are intersections of the hyperboloid with the 2-planes passing through the null line

$$
t=w, \quad x=y=z=0 .
$$

These lines spread out over the entire steady-state part of the hyperboloid, mapping to one another each entire Euclidean 3-space, given by a moment of steady-state time $t-w$, but with an exponential stretch as this time progresses. Each of these timelike lines has a separate intersection with $\mathcal{I}$, meeting it orthogonally. It seems to me that these lines represent the best analogue of a "progression of time" that we have, and that our concept of "energy" for weak-field gravitational perturbations would need to

\footnotetext{
7 This picture is to be Fig. 28.7, on p. 748 of my book The Road to Reality [28].
} 
depend upon such a notion. The fact that we are presented with a timelike conformal Killing vector, rather than an actual Killing vector may be no disaster, provided that the essential physics that we are concerned with is conformally invariant, or can be discussed in relation to conformally invariant physical processes.

In fact, according to the philosophical principles underpinning $\mathrm{CCC}$, this is indeed the case. We consider that in the extremely remote future, all rest-mass has died away (including the complete Hawking evaporation of all black holes). Technically, according to $\mathrm{CCC}$, one needs to assume that there is some mechanism (presently unknown) that causes the rest-masses of all particles to die away in the future asymptotic limit. There is no observational (or theoretical reason from the point of view of present particle physics) to expect such an eventual dying away of rest mass, but such a possibility seems not to be theoretically inconsistent. (For example, in the conventional approach to particle physics, one takes stable elementary particles to be described according to irreducible representations of the Poincaré group, where rest-mass is one of the Casimir operators. But with a positive $\Lambda$, the de-Sitter group might be more fundamentally appropriate, and rest-mass is now not a Casimir operator). In any case, it would be likely to be a good approximation to assume that the contents of the ultimate remote future of each aeon is essentially composed of massless particles. Gravitation itself is not conformally invariant, but in accordance with the discussion of Sect. 2, above (and the discussion given in Appendix B of Cycles of Time [5]) the relevant aspects of general relativity can be treated by use of conformally invariant procedures. It may well be that a conformal notion of energy, at the moment of crossover from one aeon to the next, will be adequate for what is required.

If we are to expect any analogue, for positive $\Lambda$, of the Bondi-Sachs formula for the energy carried away by gravitational energy, with we shall need to address a further issue that arises from the fact that $\mathcal{I}$ is now spacelike. With the null $\mathcal{I}$ that arises for an asymptotically flat space-time we have a clear-cut notion of the passage of retarded time, which is reflected in the fact that there is a time-direction on $\mathcal{I}$, namely that which points into the future along the null generators. Something similar occurs when a negative $\Lambda$ is present, because the timelike nature of $\mathcal{I}$ allows timelike lines to be drawn on $\mathcal{I}$, and we could consider that the passage of time is described in terms of a future evolving time parameter on each of these lines see Ashtekar and Magnon [29]. However, on the spacelike $\mathcal{I}$ that occurs with a positive $\Lambda$, any "passage of time" would carry us away from $\mathcal{I}$ itself, so we need to view things differently.

Suppose that we have a gravitational source, which emits a burst of massless radiation. If we consider a "retarded time" null hypersurfaces $\mathcal{N}_{1}$ and $\mathcal{N}_{2}$ meeting $\mathcal{I}$ in respective spacelike 2 -surfaces $\mathcal{S}_{1}$ and $\mathcal{S}_{2}$, where $\mathcal{N}_{1}$ reaches out to infinity through a region of space-time before the emitted radiation, while $\mathcal{N}_{2}$ reaches out to infinity after the radiation was emitted. This relationship is expressed, on $\mathcal{I}$, in the fact that the 2 -surface $\mathcal{S}_{2}$ lies "within" the 2-surface $\mathcal{S}_{1}$, and the emitted radiation itself makes its mark on $\mathcal{I}$ in the region of $\mathcal{I}$ that lies between $\mathcal{S}_{2}$ and $\mathcal{S}_{1}$. It would seem, therefore, that the sort of formula that needs to be aimed for would be one in which the mass/energy value assigned to $\mathcal{S}_{1}$ is equal to the corresponding mass/energy assigned to $\mathcal{S}_{2}$ plus some non-negative contribution arising from the region between the two which can be assigned the interpretation that it is the mass/energy of the radiation (gravitational plus electromagnetic, etc.). 


\section{Suggestions for an analogue of Bondi-Sachs mass, with positive $\Lambda$}

Two alternative approaches to a retarded mass/energy definition suggest themselves and I describe each of these briefly here. Each requires further investigation to see whether it provides a route to a solution of the issues raised above. The simpler of the two to state makes use of the following expression, which defines the Gaussian curvature $K+\bar{K}$ of a spacelike 2 -surface $\mathcal{S}$ :

$$
K=\sigma \sigma^{\prime}-\psi_{2}-\rho \rho^{\prime}+\Phi_{11}+R / 24
$$

In addition to the Gaussian curvature, $K$ involves an imaginary part which is a kind of extrinsic curvature quantity defined by the imbedding of $\mathcal{S}$ in the space-time (see Penrose and Rindler [23] pp. 272, 277). For the definition of the various quantities appearing in $K$, we need to choose a spin-frame $\left(o^{A}, \iota^{A}\right)$, where the flagpole of $\iota^{A}$ points along the outward null normal to $\mathcal{S}$ and that of $o^{A}$, along the inward null normal. The complex shear $\sigma$ is defined as in Sect. 2, and $\rho$ is the complex convergence (the imaginary part defining the rotation, which is zero here owing to the $o^{A}$ being normal to a 2 -surface) is given by

$$
\rho=o^{A} \iota^{B} \bar{o}^{A^{\prime}} \nabla_{A A^{\prime}} o_{B}
$$

The primed quantities $\sigma^{\prime}$ and $\rho^{\prime}$ are obtained from the unprimed ones by interchanging $o^{A}$ and $\iota^{A}$ (strictly $o^{A} \mapsto \mathrm{i} \iota^{A}, \iota^{A} \longmapsto \mathrm{i} o^{A}$ ), and $\Phi_{11}$ is the component

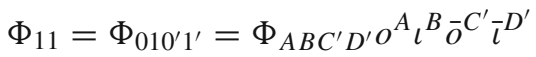

The integral of $K$ over $\mathcal{S}$, with respect to the surface area element $d \mathcal{S}$, for a compact $\mathcal{S}$ of genus $g$ is

$$
\oint_{\mathcal{S}} K d \mathcal{S}=2 \pi(1-g)
$$

and we choose $g=0$ here. The integrand splits naturally into the two parts, the first of which, $\sigma \sigma^{\prime}-\psi_{2}$, provides a manifestly conformally invariant integral

$$
Q=\oint_{\mathcal{S}}\left(\sigma \sigma^{\prime}-\psi_{2}\right) d \mathcal{S}
$$

and the second of which, $-\rho \rho^{\prime}+\Phi_{11}+R / 24$, is real, telling us that $Q$ is also real. Now the quantity $\mu_{B S}$ whose integral over $\mathcal{S}$ gives us the Bondi-Sachs mass is $(4 \pi G)^{-1}\left(\sigma \mathfrak{N}-\psi_{2}\right)$ (see Sect. 2), and $\sigma \mathfrak{N}-\psi_{2}$ is the derivative of $\sigma \sigma^{\prime}-\psi_{2}$ in the direction of $l^{a}=o^{A} \bar{o}^{A \prime}$ in the asymptotically flat case, when $\mathcal{I}$ is null. The suggestion here is that this same definition might serve also in the case when $\Lambda$ is positive, and $\mathcal{I}$ is spacelike. 
In order for this to be made precise, an appropriate normalization of the vector $l^{a}$ is needed or, equivalently, of the vector $n^{a}=\iota^{A} \bar{l}^{A^{\prime}}$ ( since $l_{a} n^{a}=1$ ) -in relation to the choice of conformal factor. What is required in the null case is that the conformal factor $\omega$ is chosen so that the metric of all cuts of $\mathcal{I}$ are unit metric spheres and that $n_{a}=\nabla_{a} \omega$ on $\mathcal{I}$. In the case $\Lambda>0$, as described in Appendix B of Cycles of Time [5], we find that the (timelike) normal vector to $\mathcal{I}$

$$
N_{a}=\nabla_{a} \omega
$$

satisfies $4 \nabla_{(a} N_{b)}=g_{a b} N_{c} N^{c}$, which in spinor terms is $\nabla_{A\left(A^{\prime}\right.} N_{\left.B^{\prime}\right) B}=0$, on $\mathcal{I}$; i.e.

$$
\nabla_{A\left(A^{\prime}\right.} \nabla_{\left.B^{\prime}\right) B} \omega=O(\omega)
$$

Moreover, it appears that we can arrange

$$
N_{a} N^{a}-\Lambda / 3=O\left(\omega^{3}\right) \text { and } \nabla_{a} N^{a}=O\left(\omega^{2}\right),
$$

and it certainly follows that

$$
\nabla_{a} N_{b}=O(\omega) \text {, i.e. } \nabla_{a} \nabla_{b} \omega=O(\omega)
$$

In accordance with this, we can choose to normalize our spin frame so that

$$
l^{a}+n^{a}=N^{a} \sqrt{6 / \Lambda}
$$

which seems to fix the energy definition in what appears to be an appropriate way, and it simplifies many of the calculations. For example, we find that with this normalization and conditions on $N^{a}$ at $\mathcal{I}$

$$
\kappa, \kappa^{\prime}, \tau, \tau^{\prime}, \rho^{\prime}+\bar{\rho}, \quad \text { and } \sigma^{\prime}+\bar{\sigma} \text { are all (at least) } O(\omega)
$$

using the (compacted) spin-coefficient formalism described in Spinors and SpaceTime $^{8}$ and the resulting equations suggest that $\Phi_{20}$ provides an appropriate definition of complex "news function" similarly to what occurs with the asymptotically flat case.

Even so, the proposal made above for the positive- $\Lambda$ version of Bondi-Sachs mass/energy is something of the nature of a "guess". More clearly motivated from the requirements of weak-field general relativity is the second proposal being put forward here, which is to make use of the ideas of quasi-local mass proposed by the author in $1982 .{ }^{9}$ In this approach, one adopts a procedure which, for linearized gravitational fields in flat space-time, would reduce the spin of the gravitational quantity $\psi_{A B C D}$ from 2 to 1 , by contracting it with a symmetric quantity $\sigma^{C D}$ satisfying the valence- $\left[\begin{array}{l}2 \\ 0\end{array}\right]$ twistor equation $\nabla^{A^{\prime}(A} \sigma^{C D)}=0$. Then the quantity $\varphi_{A B}=\psi_{A B C D} \sigma^{C D}$

\footnotetext{
8 Penrose and Rindler [23]; see also Geroch et al. [30].

9 See Penrose [31], Penrose and Rindler [24]; compare the related ideas of Goldberg [2].
} 
behaves like the spinor form of a Maxwell field whose charge integral can then be performed. For the different possible choices of $\sigma^{C D}$ we get all the different conserved sources for the linearized gravitational field, originally obtained in a different way in 1958 by Sachs and Bergmann [32]. In curved space-time, the quantity $\sigma^{C D}$ cannot generally be obtained in this way, but in relation to a specific spacelike 2-surface $\mathcal{S}$, with spherical topology, one can build up quantities like $\sigma^{C D}$ out of tensor products of pairs of valence-[ $\left.\begin{array}{l}1 \\ 0\end{array}\right]$ twistor quantities, taken relative to the 2 -surface $\mathcal{S}$, where the valence- $\left[\begin{array}{l}1 \\ 0\end{array}\right]$ twistor equation reduces to a pair of partial differential equations within $\mathcal{S}$ whose solutions define what are known as "2-surface twistors". This procedure works well for obtaining the Bondi-Sachs mass in an asymptotically flat space-time, and provides a definition that is independent of any choice of Bondi-type coordinate system. ${ }^{10}$

However, the procedure would have to be generalized appropriately if it were to apply to the asymptotically de Sitter case, as some of the equations need to be altered when a non-zero cosmological constant $\Lambda$ is present. In relation to this, a further issue that needs to be attended to is the fact that in the procedure adopted previously, when $\Lambda=0$, the quantity $\sigma^{C D}$ acts as a potential for a Killing vector, and in asymptotically flat space-time, this choice would be an asymptotic timelike Killing vector that would be appropriate to the definition of mass/energy. However, here we require a conformal Killing vector, in accordance with the discussion of Sect. 3. This tells us that some modification of the equations appropriate to the $\Lambda=0$ case is required. If this is done appropriately, a link between the two proposals being suggested here could become evident, as indeed is the case when $\Lambda=0$.

Acknowledgments The author is grateful to Ben Jeffryes for a helpful suggestion.

Open Access This article is distributed under the terms of the Creative Commons Attribution Noncommercial License which permits any noncommercial use, distribution, and reproduction in any medium, provided the original author(s) and source are credited.

\section{References}

1. Goldberg, J.N.: Strong conservation laws and equations of motion in covariant field theories. Phys. Rev. 89, 263 (1953)

2. Goldberg, J.N.: Conserved quantities at spatial and null infinity: the Penrose potential. Phys. Rev. D 41, 410 (1990)

3. Perlmutter, S., et al.: Cosmology from type Ia supernovae. Bull. Am. Astron. Soc. 29, 1351 (1997)

4. Carroll, S.M.: The cosmological constant. Living Rev. Relativ. 4 [Online Article], cited on June 2011. http://www.livingreviews.org/Articles/Volume4/2001-1carroll/

5. Penrose, R.: Cycles of Time: An Extraordinary New View of the Universe. Bodley Head, London (2010)

6. Gurzadyan, V.G., Penrose, R.: CCC-predicted low-variance circles in CMB sky and LCDM (2011). [arXiv:astro-ph/1104.5675]

7. Werhus, I.K., Eriksen, H.K.: A search for concentric circles in the 7-year WMAP temperature sky maps. ApJ 733, L29 (2011)

$\overline{10}$ This may be contrasted with the closely related approach of Goldberg [2]. 
8. Moss, A., Scott, D., Zibin, J.P.: No evidence for anomalously low variance circles on the sky. JCAP 04, 033 (2011)

9. Pais, A.: 'Subtle is the Lord...': The Science and the Life of Albert Einstein. Clarendon Press, Oxford (1982)

10. Bondi, H.: Gravitational waves in general relativity. Nature 186, 535 (1960)

11. Trautman, A.: Radiation and boundary conditions in the theory of gravitation. Bull. Acad. Polon. Sci. sér. Sci Math., Astr. Et Phys. 6, 407 (1958)

12. Trautman, A.: Conservation laws in general relativity. In: Witten, L. (ed.) Gravitation: An Introduction to Current Research, Wiley, New York (1962)

13. Bondi, H., van der Burg, M., Metzner, A.: Axisymmetric waves by multipole approximation. Proc. R. Soc. (London) A 269, 21 (1962)

14. Sachs, R.K.: Gravitational waves in general relativity VIII. Waves in asymptotically flat spacetime. Proc. R. Soc. (London) A 270, 103 (1962)

15. Sachs, R.K.: Asymptotic symmetries in gravitational theory. Phys. Rev. 128, 2851 (1962)

16. Bonnor, W.B., Rotenberg, M.A.: Gravitational waves from isolated sources. Proc. R. Soc. (Lond) A 289, 247 (1966)

17. Newman, E.T., Penrose, R.: An approach to gravitational radiation by a method of spin coefficients. J. Math. Phys. 3, 566 (1962); errata 4, 998 (1963)

18. Newman, E.T., Unti, T.W.J.: Behavior of asymptotically flat empty space. J. Math. Phys. 3, 891 (1962)

19. Streubel, M.: 'Conserved' quantities for isolated gravitational systems. Gen. Relativ. Gravit. 9, 551 (1978)

20. Penrose, R.: Conformal approach to infinity. In: DeWitt, B.S., DeWitt, C.M. (eds.) Relativity, Groups and Topology: the 1963 Les Houches Lectures, Gordon and Breach, New York (1964)

21. Penrose, R.: Zero rest-mass fields including gravitation: asymptotic behaviour. Proc. R. Soc. (London) A 284, 159 (1965)

22. Penrose, R.: Conserved quantities and conformal structure in general relativity. In: Ehlers, J. (ed) Relativity Theory and Astrophysics, Lectures in Applied mathematics, Vol. 8. American Mathematical Society, Providence (1967)

23. Penrose, R., Rindler, W.: Spinors and Space-Time, Vol. 1: Two-spinor Calculus and Relativistic Fields. Cambridge University Press, Cambridge (1984)

24. Penrose, R., Rindler, W.: Spinors and Space-time, Vol. 2: Spinor and Twistor Methods in Space-time Geometry. Cambridge University Press, Cambridge (1986)

25. Tod, K.P.: Isotropic cosmological singularities: other matter models. Class. Quantum. Gravity 20, 521 (2003)

26. Maldacena, J.M.: The large N limit of superconformal field theories and supergravity. Adv. Theoret. Math. Phys. 2, 231 (1998)

27. Witten, E.: Anti-de Sitter space and holography. Adv. Theoret. Math. Phys. 2, 253 (1998)

28. Penrose, R.: The Road to Reality: A Complete Guide to the Laws of the Universe. Vintage Books, New York (2004)

29. Ashtekar, A., Magnon, A.: Asymptotically anti-de Sitter spacetimes. Class. Quantum Gravity 1, L39-44 (1984)

30. Geroch, R., Held, A., Penrose, R.: A space-time calculus based on pairs of null directions. J. Math. Phys. 14, 874 (1973)

31. Penrose, R.: Quasi-local mass and angular momentum in general relativity. Proc. R. Soc. (London) A 381, 53 (1982)

32. Sachs, R.K., Bergmann, P.G.: Structure of particles in linearized gravitational theory. Phys. Rev. 112, 674 (1958) 